\title{
General Theorems on the Combination of Relativistic and Internal Symmetries
}

\author{
V. BERzI \\ Istituto Nazionale di Fisica Nucleare Sezione di Milano
}

Received April 29, 1968

\begin{abstract}
Theorems about the structure of a Lie algebra combining the Poincaré Lie algebra and an internal symmetry Lie algebra are derived by general algebraic methods. The physical meaning of certain mathematical results is clarified.
\end{abstract}

\section{Introduction}

Recently some interest has been devoted to the problem of combining internal and relativistic symmetries in the theory of elementary particles. The discovery of approximate internal symmetries, like $S U(3)$ symmetry for strong interacting particles, suggested the possibility of the existence of a larger symmetry group combining the internal symmetry group and the relativistic group in a non trivial way. One of the motivations was the possibility of obtaining informations about the structure of the mass spectrum from the knowledge of this larger group. Hopes in this possibility have been considerably reduced by O'Raifeartaigh's theorem; nevertheless the "combining" problem is sufficiently interesting in itself to deserve some further analysis. To be precise, we formulate the problem in terms of Lie algebras, as follows. Let $G$ be a Lie algebra containing the Lie algebra $P$ of the Poincaré group and an "internal symmetry" Lie algebra $S$ as subalgebras. Assume that $G=P+S$, direct sum of vector spaces (i.e. every element of $G$ can be uniquely written as the sum of an element of $P$ and an element of $S$ ). Then, what can be said about the structure of $G$ ? To give an answer to this question further requirements are imposed on $G$. Usually they are a) a condition on the algebraic structure of $S$ and b) an assumption which states that some elements of $[P, S]$ vanish. Under restrictions of this kind several authors [1-6] have stated "negative results" asserting that $G$ has a trivial directproduct structure: $G=P \times S$ or, at least, $G=P^{\prime} \times S^{\prime}$, where $P^{\prime}$ and $S^{\prime}$ are ideals isomorphic to $P$ and to $S$ respectively. However these results have been presented in a rather unsystematic way and with proofs based on calculations with base-dependent structure constants, which often obscure the connection between the different theorems. In this paper we shall derive several results of the type described above in a systematic 
way, by making use of the intrinsic techniques of the theory of Lie algebras [7-8]. Our method will enable to prove some new theorems and, at same time, to clarify the physical meaning of certain mathematical results.

In section II we first distinguish between two classes of negative theorems, depending on the choice of the elements of $[P, S]$ which are assumed to vanish. Then we give two general lemmas, II 1 and II 2 , and we use them to obtain two results, the lemmas II $1^{\prime}$ and II $2^{\prime}$, which give a useful tool for the derivation of the theorems of the two classes defined above. These lemmas state that $G$ has a direct-product structure if one assumes that $P$ or $S$ is an ideal of $G$. Finally the lemma II 3 gives some information on the structure of a Lie algebra containing the Poincaré Lie algebra as an ideal.

In section III we give a number of theorems of the first class defined in section II. Theorem III 1 states that $G=P \times S$ under assumptions which are similar but less restrictive than those of McGlinn theorem [1]. Only the elements of $P$ which generate the pure Lorentz transformations are assumed to commute with $S$ and the condition of semi-simplicity on $S$ is replaced by the more general condition $[S, S]=S$. These assumptions can be weakened by introducing the concept of regular element of a Lie algebra [7]; if we retain the preceding condition on $S$ and we suppose that a regular element of the Lorentz subalgebra $L$ of $P$ commutes with $S$, we have $G=P \times S^{\prime}$ (with $S^{\prime}$ isomorphic to $S$ but not necessarily coinciding with $S$ ). This is proved by the theorem III 2 . The elements of $L$ which generate the space rotations or the pure Lorentz transformations are regular elements of $L$. Thus the result $G=P \times S^{\prime}$ under the assumption that one of these elements commutes with $S$, can be obtained as a corollary of the preceding theorem (corollary III 1). A Lie algebra $E$ containing $P$ as a subalgebra can be written in the form $E=P+A$, where $A$ is a subspace having zero intersection with $E$. If we assume that the elements of a set of generators of $L$ or a regular element of $L$ commute with $A$, we arrive to the conclusion that $P$ is an ideal of $E$. Then by using lemma II 3, we can prove that, under certain conditions, $E$ has a direct-product structure, with $P$ as a factor. Finally, theorem III 5 gives a result which can be considered complementary to the one of McGlinn. It states that $G=P^{\prime} \times S$ with $P^{\prime}$ isomorphic to $P$, under assumption that $S$ is semisimple and that the elements of the translations ideal of $P$ commute with $S$.

In section IV we give some theorems of the second class defined in section II. The more important results are the theorems IV 1 and IV 4. In both of them a single element $q$ of $S$ is assumed to commute with $P$ and $S$ supposed to be semisimple. In theorem IV $1 q$ is required to be regular in $S$. This condition is discussed from a physical viewpoint, with 
reference to the example of the $S U(3)$ symmetry. In this way it is clarified the meaning of theorem IV 4 in which a less restrictive condition is imposed on $q$.

\section{Some Preliminary Results}

We start by introducing some notations and definitions used in this paper. We use the notation $A \approx B$ for $A$ isomorphic to $B$. If $A$ is a Lie algebra and $A_{1}$ and $A_{2}$ are subalgebras of $A$, we write $A=A_{1}+A_{2}$ (direct sum of vector spaces) or $A=A_{1}+A_{2}$ (d.s.) or simply $A_{1}+A_{2}$, if the vector space $A$ is the direct sum of the subspaces $A_{1}$ and $A_{2}$ (this implies $A_{1} \cap A_{2}=0$ ). If in addition $A_{1}$ and $A_{2}$ are ideals of $A$, we say that $A$ is the direct product of $A_{1}$ and $A_{2}$ and we write $A=A_{1} \times A_{2}$. We denote by $\operatorname{der} A$ the Lie algebra of derivations of $A$ and by ad the adjoint mapping of $A$; if $B$ is a subalgebra of $A$ the adjoint mapping of $B$ will be denoted by ad ${ }^{B}$; if $B$ is an ideal and $a \in A$ we denote by $\operatorname{ad}_{B}$ a the restriction of ad $a$ to $B$ (so that if $b \in B$ we have $\operatorname{ad}^{B} b=\operatorname{ad}_{B} b$ ). All Lie algebras will be over the real field and finite-dimensional, unless explicitely stated otherwise. Throughout the paper $G$ will denote a Lie algebra containing the Poincaré Lie algebra $P$ and an internal Lie algebra $S$ as subalgebras in such way that:

$$
G=P+S \text { (d.s.) }
$$

The structure of $P$ is well known; $P=L+T$ (d.s.) where $L$ is the Lie algebra of the Lorentz group and $T$ a four-dimensional abelian ideal. $P$ is spanned by a set of elements $m_{\mu \nu} \in L(\mu, v=0, \ldots, 3)$ and $t_{\mu} \in T$ $(\mu=0, \ldots, 3)$ where the $m_{i j}(i, j=1,2,3)$ generate the rotations in the plane $(i, j)$, the $m_{0 k}(k=1,2,3)$ generate the pure Lorentz transformations along the $k$-axis and the $t_{\mu}$ generate the space-time translations. For these elements we have:

$$
\begin{aligned}
{\left[m_{\mu \nu}, m_{\varrho \sigma}\right] } & =g_{\mu \sigma} m_{\nu \varrho}+g_{\nu \varrho} m_{\mu \sigma}-g_{\mu \varrho} m_{\nu \sigma}-g_{v \sigma} m_{\mu \varrho} \\
{\left[m_{\mu \nu}, t_{\varrho}\right] } & =g_{\mu \varrho} t_{\nu}-g_{\nu \varrho} t_{\mu} \\
{\left[t_{\mu}, t_{v}\right] } & =0 \\
\left(g_{\mu \nu}\right) & =\operatorname{diag}\{1,-1,-1,-1\} .
\end{aligned}
$$

As explained in the introduction, negative results can be obtained under assumptions which state the vanishing of the elements of a subset of $[P, S]$. These assumptions can be divided in two classes as follows.

Class $1 .[p, S]=0$ where $p$ belongs to a subset of $P$.

Class 2. $[P, s]=0$ where $s$ belongs to a subset of $S$ (or of $S^{C}$, the complexification of $S$; see section IV).

The following lemmas give a general tool for the derivation of the negative theorems which hold under the assumptions of class 1 (lemma 1') and of class 2 (lemma $2^{\prime}$ ). 
Lemma 1. Let $E$ be a Lie algebra and let $E=A+B(d . s$.$) , where A$ is an ideal and $B$ a subalgebra. Let $\varphi$ be the homomorphism $b \rightarrow \operatorname{ad}_{A} b$ of $B$ into der $A$. If

$$
\varphi=\operatorname{ad}^{A} \circ \psi
$$

where $\psi$ is a homomorphism of $B$ into $A$, then $E=A \times B^{\prime}$ and $B^{\prime} \approx B$.

Proof. It suffices to prove that there exists an ideal $B^{\prime} \subset E$ such that $E=A+B^{\prime}$ (d.s.); in fact this implies $B^{\prime} \approx E / A \approx B$. Let $B^{\prime}$ be the set of elements of the form $-\psi(b)+b, b \in B$. It is clear that $B^{\prime}$ is a subspace such that $E=A+B^{\prime}$ and $B^{\prime} \cap A=0$. Next, let $x$ be an arbitrary element in $E$; we can write $x=a+b_{1}, a \in A, b_{1} \in B$, then, for $b \in B$, we have

$$
[x,-\psi(b)+b]=-[a, \psi(b)]-\left[b_{1}, \psi(b)\right]+[a, b]+\left[b_{1}, b\right] .
$$

From (3) one obtains $[a, b]=[a, \psi(b)]$ and $\left[b_{1}, \psi(b)\right]=\psi\left(\left[b_{1}, b\right]\right)$; thus $[x,-\psi(b)+b]=-\psi\left(\left[b_{1}, b\right]\right)+\left[b_{1}, b\right] \in B^{\prime}$ and $B^{\prime}$ is an ideal.

Lemma 2 is an elementary result of the theory of Lie algebras (ref. [7] Ch. I proposition 3).

Lemma 2. If $K$ is a complete ideal in the Lie algebra $E$ then $E=K \times H$ where $H$ is the centraliser of $K$.

We now use the preceding lemmas to obtain two results on the structure of $G=P+S$. To this aim, we need some information on the structure of $\operatorname{der} P$. On can prove [9] that every derivation $D \in \operatorname{der} P$ is of the form

$$
D=\operatorname{ad} p(D)+\alpha(D) \pi
$$

where $p(D) \in P, \alpha(D)$ is a real number and $\pi$ is the projection onto $T$ with kernel $L$. Further if $D_{1}, D_{2} \in \operatorname{der} P$ then

$$
\begin{aligned}
& \alpha\left(D_{1}+D_{2}\right)=\alpha\left(D_{1}\right)+\alpha\left(D_{2}\right) \\
& \alpha\left(\left[D_{1}, D_{2}\right]\right)=0 .
\end{aligned}
$$

Lemma $1^{\prime}$. If $P$ is an ideal of $G=P+S$ and $S$ coincides with its derived algebra i.e. $[S, S]=S$, then $G=P \times S^{\prime}$ with $S^{\prime} \approx S$.

Proof. By lemma 1 it suffices to prove that the homomorphism $\varphi: x \rightarrow \operatorname{ad}_{P} x$ of $S$ into der $P$ is of the form (3). Since $\operatorname{ad}_{P} x \in d e r P$, can write $\varphi(x)=\operatorname{ad}^{P} p(\varphi(x))+\alpha(\varphi(x)) \pi$. But $[S, S]=S$ implies that every $x \in S$ can be written in the form $x=\Sigma\left[x_{i}, y_{i}\right], x_{i}, y_{i} \in S$. Hence from (5) it follows that $\alpha(\varphi(x))=0$ and this shows that $\varphi$ is a homomorphism of $S$ into $\operatorname{ad}^{P} P$. Since the center of $P$ is zero, $\operatorname{ad}^{P}$ is an isomorphism of $P$ onto $\operatorname{ad}^{P} P$. Hence $\left(\operatorname{ad}^{P}\right)^{-1}$ exists and is an isomorphism of $\operatorname{ad}^{P} P$ onto $P$. Then $\psi=\left(\operatorname{ad}^{P}\right)^{-1} \circ \varphi$ is a homomorphism of $S$ into $P$; thus $\varphi=\operatorname{ad}^{P} \circ \psi$. This completes the proof.

Remark 1. Lemmas 1 and $\mathbf{l}^{\prime}$ are equivalent to two results of M. Flato and D. Sternheimer [9], which are stated in terms of Lie algebras extensions. 
Remark 2. The condition $[S, S]=S$ includes $S$ semisimple, but is more general; for instance one has $[P, P]=P$.

Lemma 2'. If $S$ is a semisimple ideal of $G=P+S$ then $G=P^{\prime} \times S$ with $P^{\prime} \approx P$.

Proof. A semisimple Lie algebra has zero center and its derivations are all inner, hence it is complete. Then from lemma 1 it follows $G=P^{\prime} \times S$ and from $P \approx G / S, P^{\prime} \approx G / S$, it follows $P^{\prime} \approx P$.

Lemma 2 asserts that a Lie algebra containing a complete ideal has a direct product structure, with this ideal as a factor. Now $P$ has zero center but it not complete, as for instance $\pi$ in (4) is not an inner derivation; for a Lie algebra containing $P$ as an ideal we can only give the following weaker result, which we shall use in section III.

Lemma 3. Let $P$ be an ideal in a Lie algebra $E$, then $E=P+A$ (d.s.) where $A$ is a subalgebra.

Proof. For every $x \in E \operatorname{ad}_{P} x \in \operatorname{der} P$; hence $\operatorname{ad}_{P} x=\operatorname{ad}_{P} p+\alpha \pi$ where $p \in P, \alpha$ is a real number and $\pi$ is defined as in (4). Let $A$ be the set of elements $a \in E$ such that $\operatorname{ad}_{P} a=\alpha \pi$ for some $\alpha$. It is clear that $A$ is a subalgebra. Next, let $y \in A \cap P$; then $y=l+t, l \in L, t \in T$ and $[y, u]=\alpha u$ for every $u \in T$; this is impossible unless $\alpha=0$ and $l=0$. Then $y$ is in the center of $P$ which is zero. Thus $A \cap P=0$.

Now let $x$ be arbitrary in $E$. Then $\operatorname{ad}_{P}(x-p)=\alpha \pi$ for some $p \in P$. This implies $a=x-p \in A$, which completes the proof.

\section{A First Class of Negative Theorems}

In this section we derive a number of negative theorems, which hold under the assumptions of class 1 described in section II.

Consider the following elements of $L: J_{k}^{\prime}=m_{0 k}(k=1,2,3)$ where the $m_{0 k}$ are defined as in section II. Then the following theorem generalizes the result of McGlinn [1].

Theorem 1. If, in $G=P+S,[S, S]=S$ and

then $G=P \times S$.

$$
\left[J_{k}^{\prime}, S\right]=0, \quad k=1,2,3
$$

Proof. From (11.2) it follows that the $J_{k}^{\prime}$ are a set of generators for $L$. Then $\left[J_{k}^{\prime}, S\right]=0$ implies $[L, S]=0$. Again from (11.2) it follows that every $t \in T$ can be written in the form $t=[l, u], l \in L, u \in T$.

If $s \in S$ from the Jacobi identity and from $[L, S]=0$ we obtain $[s, t]=-[l,[u, s]]$; now $[u, s]$ can be decomposed in the form: $[u, s]$ $=l_{1}+t_{1}+s_{1}, l_{1} \in L, t_{1} \in T, s_{1} \in S$. Hence $[s, t]=-\left[l, l_{1}\right]-\left[l, t_{1}\right] \in P$.

Thus $P$ is an ideal in $G$ and $G=P \times S^{\prime}, S^{\prime} \approx S$ by lemma II $1^{\prime}$. Next, let $s$ be arbitrary in $S$; then $s=p+s^{\prime}, p \in P, s^{\prime} \in S^{\prime}$; from $[L, S]=0$ and $\left[P, S^{\prime}\right]=0$ we have $[p, l]=0$ for every $l \in L$. Hence $p=0$ and $s \in S^{\prime}$; thus $S \leqq S^{\prime}$, but $S \approx S^{\prime}$ so that $S=S^{\prime}$ and $G=P \times S$. 
One can prove that $G$ has a direct product structure with $P$ as a factor under the weaker assumption $[r, S]=0$, where $r$ is an element of $L$, provided that $r$ is regular in $L$. To obtain this result we require the following

Lemma 1. Let $r$ be a regular element of $L$. Then there exists in $L$ a basis $\left\{e_{1}, e_{2}, e_{3}, e_{1}^{\prime}, e_{2}^{\prime}, e_{3}^{\prime}\right\}$ such that $r=e_{3}$ and;

$$
\left[e_{i}, e_{j}\right]=\varepsilon_{i j k} e_{k}, \quad\left[e_{i}, e_{j}^{\prime}\right]=\varepsilon_{i j k} e_{k}^{\prime}, \quad\left[e_{i}^{\prime}, e_{j}^{\prime}\right]=-\varepsilon_{i j k} e_{k}
$$

(where $\varepsilon_{i j k}$ is the totally antisymmetric symbol and the sum convention over repeated indices is adopted).

Proof. We recall that an element $x$ of a Lie algebra $E$ (over an arbitrary field) is called regular if the Fitting null component of $E$ relative to $\operatorname{ad} x$, i.e. the subspace $\left\{y \in E \mid(\operatorname{ad} x)^{k} y=0\right.$ for some $\left.k\right\}$ has minimal dimension. Let $E$ be a complex Lie algebra. We shall denote by $E^{R}$ the real Lie algebra obtained from $E$ by restriction of the base field (for this notion see [8] Ch. III). It is not difficult to see that an element $x \in E$ is regular if and only if it is regular in $E^{R}$. Now it is well known that $L$ is isomorphic into $\operatorname{sl}(2, C)^{R}$; hence if $\varphi$ is an isomorphism of $L$ onto $s l(2, C)^{R}$, then $h=\varphi(r)$ is regular in $\operatorname{sl}(2, C)^{R}$ and in $\operatorname{sl}(2, C)$. By definition $k=2 i h$ is also regular in $s l(2, C)$. Then $k$ belongs to a basis $\{e, f, k\}$ such that:

$$
[k, e]=2 e, \quad[k, f]=-2 f, \quad[e, f]=k .
$$

Now we set $e^{\prime}=i e, f^{\prime}=i f, k^{\prime}=i k$ and

$$
\begin{array}{ll}
\bar{e}_{1}=\frac{1}{2}(e-f), \quad \bar{e}_{2}=-\frac{1}{2}\left(e^{\prime}+f^{\prime}\right), & \bar{e}_{3}=-\frac{1}{2} k^{\prime}=h, \\
\bar{e}_{1}^{\prime}=\frac{1}{2}\left(e^{\prime}-f^{\prime}\right), \quad \bar{e}_{2}^{\prime}=\frac{1}{2}(e+f), \quad \bar{e}_{3}^{\prime}=\frac{1}{2} k .
\end{array}
$$

One can verify that the elements $\bar{e}_{i}$ and $\bar{e}_{j}^{\prime}$ form a basis of $s l(2, C)^{R}$ and satisfy (2). Then $L$ has a basis $e_{i}=\varphi^{-1}\left(\bar{e}_{i}\right), e_{j}=\varphi^{-1}\left(\bar{e}_{j}\right),(i, j=1,2,3)$ with multiplication table (2) and $e_{3}=r$ as required.

We are now in a position to obtain the result mentioned above.

Theorem 2. If, in $G=P+S,[S, S]=S$ and

$$
[r, S]=0
$$

where $r \in L$ is regular, then $G=P \times S^{\prime}, S \approx S^{\prime}$.

Proof. By lemma II $1^{\prime}$ it suffices to prove that $P$ is an ideal in $G$. Let $\left\{e_{1}, e_{2}, e_{3}, e_{1}^{\prime}, e_{2}^{\prime}, e_{3}^{\prime}\right\}$ be a basis of $L$ with multiplication table (2) and $e_{3}=r$. We shall first show that if $s \in S$ then $\left[s, e_{k}\right] \in P,(k=2,3)$ and $\left[s, e_{k}^{\prime}\right] \in P$. By (2), (3) and the Jacoby identity we have: $\left[s, e_{1}\right]=\left[s,\left[e_{2}, e_{3}\right]\right]$ $=-\left[e_{3},\left[s, e_{2}\right]\right] ;$ since we can write $\left[s, e_{2}\right]=p+s_{1}, p \in P, s_{1} \in S$, by (3) we have $\left[s, e_{1}\right] \in P$. In a similar way we obtain $\left[s, e_{2}\right],\left[s, e_{1}^{\prime}\right],\left[s, e_{2}^{\prime}\right] \in P$; hence $\left[s, e_{3}^{\prime}\right]=\left[e_{1}^{\prime},\left[s, e_{2}\right]\right]+\left[\left[s, e_{1}^{\prime}\right], e_{2}\right] \in P$. Thus $[S, L] \leqq P$; since every $t \in T$ can be written in the form $t=[l, u], l \in L, u \in T$, it follows $[s, t] \in P$ for every $s \in S$ and $t \in T$. Then $P$ is an ideal. 
The concept of regular element is purely mathematical; in view of this it will be worth to give a result similar to the preceding one, but formulated in terms of elements of $L$, which have a more direct physical meaning.

Corollary 1. Let $m_{\mu \nu} \in L(\mu, v=0,1,2,3)$ be defined as in section II. If, in $G=P+S,[S, S]=S$ and

then $G=P \times S^{\prime}, S^{\prime} \approx S$.

$$
\left[m_{\mu \nu}, S\right]=0
$$

Proof. Let us pose $J_{i}=m_{j k}(i, j, k$ cyclic permutation of $1,2,3)$ and $J_{k}^{\prime}=m_{0 k}(k=1,2,3)$.

It suffices to prove that these elements are regular in $L$. By (II 22) we have:

$$
\left[J_{i}, J_{j}\right]=\varepsilon_{i j k} J_{k}, \quad\left[J_{i}, J_{j}^{\prime}\right]=\varepsilon_{i j k} J_{k}^{\prime}, \quad\left[J_{i}^{\prime}, J_{j}^{\prime}\right]=-\varepsilon_{i j k} J_{k} .
$$

Let $\varphi$ be an isomorphism of $L$ onto $s l(2, C)^{R}$ and $\bar{J}_{i}=\varphi\left(J_{i}\right), \bar{J}_{i}^{\prime}=\varphi\left(\bar{J}_{i}\right)$. If we pose $e=\bar{J}_{1}+i \bar{J}_{2}, f=-\bar{J}_{1}+i \bar{J}_{2}$ and $h=i \bar{J}_{3}$, then $\{e, f, h\}$ is a basis of $\operatorname{sl}(2, C)$ and:

$$
[h, e]=e, \quad[h, f]=-f, \quad[e, f]=2 h .
$$

If $x \in \operatorname{sl}(2, C)$ we can write $x=\alpha e+\beta f+\gamma h$. Hence by $(6)(\operatorname{ad} h)^{m} x=0$ implies $\alpha e+(-1)^{m} \beta f=0$; thus $\alpha=0$ and $\beta=0$. This shows that the Fitting null component of $\operatorname{sl}(2, C)$ relative to ad $h$ is one-dimensional; then $h$ is regular in $s l(2, C)$ and consequently in $s l(2, C)^{R}$. The same holds for $\bar{J}_{3}=-i h$, hence $J_{3}=\varphi^{-1}\left(\bar{J}_{3}\right)$ is regular in $L$. The regularity of the other elements can be proved in a similar way.

If one examines the proofs of the theorems 1 and 2 , one can observe that, to obtain that $P$ is an ideal in $G$, the assumption " $S$ is a subalgebra of $G$ " is not necessary; the result holds as well if $S$ is assumed to be a subspace of $G$. This remark can be applied to the study of the problem of imbedding the Poincaré algebra into a larger Lie algebra. This can be considered as a slight generalization of the problem discussed in the introduction. We are concerned with a Lie algebra $E$ containing $P$ as subalgebra. Obviously $E$ can always written in the form:

$$
E=P+A
$$

where $A$ is a subspace and $P \cap A=0$. Under assumptions on the elements of $[P, A]$, which are similar to those of theorems 1 and 2 , we can derive the following results (compare reference [2]).

Theorem 3. Let $P$ be a subalgebra of a Lie algebra $E$ and $A$ a subspace of $E$ such that (7) holds. If

$$
\left[J_{j}^{\prime}, A\right]=0, \quad i=1,2,3
$$

then a) $P$ is an ideal and $E=P+A^{\prime}$ (d.s.), where $A^{\prime}$ is a subalgebra. b) $A \cap P=0$ and $A$ is a subalgebra. c) if $A=[A, A]$ then $G=P \times A$. 
Proof. a) follows from the remark above and lemma II 3. Next, (8) implies $[L, A]=0$, hence $A \cap P=0$. If $a, b \in A[[a, b], L]=0$ and we can write $[a, b]=p+c, p \in P, c \in A$. Hence $[p, L]=0$, which implies $p=0$ and $[a, b] \in A$. This proves $b$ ). Finally if $[A, A]=A$ all the hypotheses of theorem 1 are satisfied and c) follows.

Theorem 4. Let $P$ be a subalgebra of the Lie algebra $E$ and $A$ a subspace such that (7) holds and $A \cap P=0$. If

$$
[r, A]=0
$$

where $r$ is a regular element of $L$, then a) $P$ is an ideal and $E=P+A^{\prime}$ (d.s.), where $A^{\prime}$ is a subalgebra, $b$ ) let $\alpha$ be the projection onto $A$ with kernel $P:$ then $A$ equipped with the composition law $(a, b) \rightarrow \alpha[a, b]$ becomes a Lie algebra isomorphic to $A^{\prime}$; c) if $\left[A^{\prime}, A^{\prime}\right]=A^{\prime}$ then $G=P \times A^{\prime \prime}$, $A^{\prime \prime} \approx A^{\prime} \approx A$.

Proof. That $P$ is an ideal can be proved with the method used for theorem 2. Then a) follows from lemma II 3. The mapping $(a, b)$ $\rightarrow \alpha[a, b]$ is clearly a bilinear mapping of $(A, A)$ into $A$ and $\alpha[a, a]=0$ for every $a \in A$. Let us pose $\{a, b\}=\alpha[a, b]$ for $a, b \in A$. Since $P$ is an ideal and $(1-\alpha)[b, c] \in P$ we have $\alpha[a,(1-\alpha)[b, c]]=0(a, b, c \in A)$. Hence $\{a,\{b, c\}\}=\alpha[a, \alpha[b, c]]=\alpha[a,[b, c]]$. Then the Jacobi identity for \{\} is a consequence of the Jacobi identity for [ ]; thus the first assertion of $b$ ) is proved. Next, let $\pi$ be the projection onto $A^{\prime}$ with kernel $P$. The restriction of $\pi$ onto $A$ is clearly an isomorphism of the vector space $A$ on the vector space $A^{\prime}$. Since $\pi P=0$, we have:

$$
\begin{aligned}
\pi \alpha[a, b] & =\pi[a, b]=\pi[\pi a+(1-\pi) a, \pi b+(1-\pi) b] \\
& =\pi[\pi a, \pi b]=[\pi a, \pi b]
\end{aligned}
$$

which implies $A \approx A^{\prime}$; this completes the proof of b). Finally c) follows from lemma II $\mathbf{1}^{\prime}$.

We conclude this section by giving a theorem which is in some sense complementary to the one of McGlinn [1]. To obtain this result we require the following lemma 2 , due to C. Itzykson [3]. The proof we shall give is much simpler than Itzykson's proof and can well illustrate the power of the intrinsic methods in dealing with Lie algebras.

Lemma 2. Let $P$ be a subalgebra of a Lie algebra $E$ and $A$ a subspace of $E$ such that $E=P+A$ and $A \cap P=0$. If

$$
[T, A]=0
$$

then a) $T$ is an ideal of $E: b$ ) let $\varphi$ be the natural homomorphism of $E$ onto $E / T$, then $\varphi(A)$ is an ideal of $E / T$ and if it is semisimple one has $E=P^{\prime} \times A^{\prime}$, where $P^{\prime} \approx P$ and $A^{\prime}$ is a semisimple ideal.

Proof. a) Let $x$ be an arbitrary element of $E$; we can write $x=l$ $+u+a, l \in L, u \in T, a \in A$; hence $[x, t]=[l, t] \in T$ for every $t \in T$ and $T$ is an ideal. b) We prove first that $A+T$ is an ideal of $E$. It will 
suffice to show that $[x, a] \in A+T$ for $a \in A$ and $x \in E$; we write $x=l+u+b, l \in L, u \in T, b \in A$ so that $[x, a]=[l, a]+[b, a]$. By the Jacobi identity we have $[t,[b, a]]=0$ for every $t \in T$. Hence if $[b, a]$ $=c+m$ with $c \in A+T$ and $m \in L$ we obtain $[m, t]=0$; this implies $m=0$ and $[b, a] \in A+T$.

In the same way, since $[t,[l, a]]=-[l,[a, t]]-[a,[t, l]]=0$ for every $t \in T$, we obtain $[l, a] \in A+T$. Thus $A+T$ is an ideal of $E$; then $\varphi(A+T)$ is an ideal of $E / T$. But $\varphi(A+T)=\varphi(A)$, hence $\varphi(A)$ is an ideal of $E / T$. Now let us suppose that $\varphi(A)$ is semisimple. We observe that $T$ is the radical of the Lie algebra $A+T$. In fact $T$ is an abelian ideal, so that if $R$ is the radical, then $R \supseteqq T$; the restriction of $\varphi$ onto $A+T$ is a homomorphism of $A+T$ onto $\varphi(A)$, hence $\varphi(R)$ is an ideal of $\varphi(A)$; but $\varphi(R)$ is solvable as homomorphic image of a solvable Lie algebra. Hence the semisimplicity of $\varphi(A)$ implies $\varphi(R)=0$; it follows $R \leqq T$ and $R=T$ as required. Then by Levi's theorem $A+T$ contains a semisimple subalgebra $A^{\prime}$ such that $A+T=A^{\prime}+T$ (d.s.); from $[A, T]=0$ it follows $\left[A^{\prime}, T\right]=0$ since if $a^{\prime} \in A^{\prime}$ we can write $a^{\prime}=a+t$, $a \in A, t \in T$. Now we recall that if $F$ is a subalgebra of a Lie algebra $E$, then a derivation $D$ of $F$ into $E$ is a linear mapping of $F$ into $E$ such that $D\left[f_{1}, f_{2}\right]=\left[D f_{1}, f_{2}\right]+\left[f_{1}, D f_{2}\right]$ for every $f_{1}, f_{2} \in F$. As $A+T$ is an ideal of $E$, it follows that $\operatorname{ad}_{A}, l, l \in L$, is a derivation of $A^{\prime}$ into $A^{\prime}+T$. Since $A^{\prime}$ is semisimple this derivation can be extended to an inner derivation of $A^{\prime}+T$ (reference [7] Ch. III Theorem 9). This means that there exists an element $x \in A^{\prime}+T$ such that $[l, a]=[x, a]$ for every $a \in A^{\prime}$. Since $\left[A^{\prime}, T\right]=0$ this implies $[l, a] \in A^{\prime}$. Thus $A^{\prime}$ is an ideal of $E$ and by lemma II $2 E=P^{\prime} \times A^{\prime}$, where $P^{\prime} \approx P$.

Theorem 5. If the subalgebra $S$ in $G=P+S$ is semisimple and $[S, T]=0$, then $G=P^{\prime} \times S, P^{\prime} \approx P$.

Proof. Let $\varphi$ be the natural homomorphism of $G$ onto $G / T$. Then $\varphi(S)$ is isomorphic to $S$ hence semisimple. Then the proof of the preceding lemma shows that $S$ is an ideal in $G$. Hence by lemma II $2^{\prime} G=P^{\prime} \times S$ with $P^{\prime} \approx P$.

\section{A Second Class of Negative Theorems}

In this section we give several results on the structure of $G=P+S$, which hold under assumptions of class 2 defined in section II. We shall use the concept of complexification of a real Lie algebra. For a definition we refer to reference [8] (Ch. III § 6); we shall only give here a number of consequences of this definition. Let $E$ be a real Lie algebra. We shall denote by $E^{C}$ its complexification; $E$ can be identified with a subset of $E^{C}$. With this identification the elements of $E^{C}$ can be written in the form $x+i y, x, y \in E ; x+i y=0$ implies $x=0$ and $y=0$. If $E_{1}$ is a subalgebra or an ideal of $E$, then $E_{1}^{C}$ is a subalgebra or an ideal of $E^{C}$. If $E=E_{1}+E_{2}$, direct sum of vector spaces, then $E^{C}=E_{1}^{C}+E_{2}^{C}$, direct 
sum of vector spaces. The Lie algebra $S$ is semisimple if and only if $S^{C}$ is semisimple. Finally an element $x \in E$ is regular in $E$ if and only if it is regular in $E^{C}$.

Theorem 1. If the subalgebra $S$ in $G=P+S$ is semisimple and

$$
[q, p]=0
$$

where $q$ is a regular element of $S$, then $G=P \times S$.

Proof. We have $G^{C}=P^{C}+S^{C}$ (d.s.) and $q$ is regular in $S^{C}$. Hence the Fitting null component of $S^{C}$ relative to ad $q$ is a Cartan subalgebra $K$ containing $q$ and $S^{C}$ can be decomposed in the form

$$
S^{C}=K+\sum_{\alpha \in R} S_{\alpha}
$$

where $R$ is the set of non-zero roots of $K$, and $S_{\alpha}$ is the root subspace relative to $\alpha$. If $e_{\alpha}$ is a non-zero element of $S_{\alpha}$, by definition we have $\left[q, e_{\alpha}\right]=\alpha(q) e_{\alpha}$ and $\alpha(q) \neq 0$ for every $\alpha \in R$. Hence we can write:

$$
e_{\alpha}=\frac{1}{\alpha(q)}\left[q, e_{\alpha}\right]
$$

and by the Jacobi identity and (1):

$$
\left[p, e_{\alpha}\right]=-\frac{1}{\alpha(q)}\left[q,\left[e_{\alpha}, p\right]\right]
$$

for every $p \in P^{C}$. Then if one writes $\left[e_{\alpha}, p\right]=p^{\prime}+s, p^{\prime} \in P^{C}, s \in S^{C}$, one sees that $\left[p, e_{\alpha}\right] \in S^{C}$.

Now we recall that if $K^{*}$ denotes the dual space of $K$, we have an isomorphism $\varrho \rightarrow h_{\varrho}$ of $K^{*}$ onto $K$, where $h_{\varrho}$ is defined by the condition $B\left(k, h_{\varrho}\right)=\varrho(k)$ for every $k \in K, B$ being the Killing form of $S^{C}$.

Further, the elements $h_{\alpha}, \alpha \in R \leqq K^{*}$, span $K$ and one can choose $e_{\alpha} \in S_{\alpha}$ so that $h_{\alpha}=\left[e_{\alpha}, e_{-\alpha}\right]$. Then $\left[p, h_{\alpha}\right] \in S^{C}$ for every $p \in P^{C}, \alpha \in R$. Since the elements $h_{\alpha}, e_{\alpha}, \alpha \in R$ span $S^{C}$ we conclude that $S^{C}$ is an ideal of $G^{C}$. Then by lemma II 2 we have $G^{C}=S^{C} \times P^{\prime}$ with $P^{\prime} \approx P^{C}$. Hence every $p \in P^{C}$ can be uniquely decomposed in the form $p=p^{\prime}+s$ with $p^{\prime} \in P^{\prime}$ and $s \in S^{C}$. Since $\left[P^{\prime}, S^{C}\right]=0$ and $[q, P]=0$ we have ad $q(s)=0$, so that $s \in K$. Now let $\pi$ be the projection onto $S^{C}$ with kernel $P^{\prime}$; since $P^{\prime}$ and $S^{C}$ are ideals $\pi$ is a homomorphism of $G^{C}$ onto $S^{C}$; further $\pi P^{C} \leqq K$. It follows that the restriction of $\pi$ onto the subalgebra $L^{C}$ of $P^{C}$ is a homomorphism of $L^{C}$ into $K$. Since $L^{C}$ is semisimple and $K$ is abelian $\pi L^{C}=0$, so that $L^{C} \leqq P^{\prime}$ and $\left[S^{C}, L^{C}\right]=0$. This implies $[L, S]=0$. Hence by theorem III $1 G=P \times S$.

By the preceding theorem, or by using similar methods of proof, one can obtain several related results, for instance all those of reference [4]. We shall give only two examples

Theorem 2. If the subalgebra $S$ in $G=P+S$ is semisimple and

$$
[P, K]=0 \text {, }
$$

where $K$ is a Cartan subalgebra of $S^{C}$, then $G=P \times S$. 
Proof. Every Cartan subalgebra of a Lie algebra over an algebraically closed field of characteristic zero contains a regular element (reference [7] Ch. IX theorem 3, remarks). Hence $K$ contains a regular element $q$ such that $[q, P]=0$. Thus by theorem 1 it follows $G=P \times S$.

Theorem 3. Let $S$ be a semisimple subalgebra of $G=P+S, K a$ Cartan subalgebra of $S^{C}$ and $S_{\alpha}$ the root subspaces. If the condition;

$$
\left[r, S_{\alpha}\right]=0 \text {. }
$$

Where $r$ is a regular element of $L$, holds for every root $\alpha$ then $G=P \times S^{\prime}$, $S^{\prime} \approx S$.

Proof. Let $h_{\alpha} \in K$ be defined as in the proof of theorem 1. We can choose $e_{\alpha} \in S_{\alpha}$ such that $h_{\alpha}=\left[e_{\alpha}, e_{-\alpha}\right]$. By (4) we have $\left[r, h_{\alpha}\right]=0$. Since the elements $e_{\alpha}$ and $h_{\alpha}$ span $S^{C}$, it follows $\left[r, S^{C}\right]=0$ and $[r, S]=0$. Then by theorem III $2 G=P \times S^{\prime}$ with $S \approx S^{\prime}$.

Remark. Under the assumption that the $S_{\alpha}$ commute with the elements of a set of generators of $L$ (for instance with the $J_{i}^{\prime}$ ) one obtains in a similar way the stronger result $G=P \times S$.

Condition (1) of theorem 1 has not an easy interpretation from a physical viewpoint as $q$ is restricted to be regular. This is illustrated by the following considerations. Suppose $S$ is isomorphic to $S U(3)$. Then $S^{C}$ is a complex Lie algebra of type $A_{2}$. Consequently as is well known, $S^{C}$ contains a basis $\left\{e_{i}\right\}(i=1,2, \ldots, 8)$ with multiplication table

$$
\left[e_{i}, e_{j}\right]=f_{i j k} e_{k}
$$

where the $f_{i j k}$ are odd under permutation of any two indices and the non zero elements are:

$$
\begin{aligned}
& f_{123}=1, \quad f_{147}=\frac{1}{2}, \quad f_{156}=-\frac{1}{2}, \quad f_{246}=\frac{1}{2}, \quad f_{257}=\frac{1}{2} \\
& f_{345}=\frac{1}{2}, \quad f_{367}=-\frac{1}{2}, \quad f_{458}=\frac{\sqrt{3}}{2}, \quad f_{678}=\frac{\sqrt{3}}{2} .
\end{aligned}
$$

We note that the set of linear combinations of the $e_{i}$ with real coefficients is a real Lie algebra which can be identified with $S$. From (5) and (6) we see that the elements $i e_{k}$ have the same commutation rules as the elements $\left\{F_{i}\right\}(i=1,2, \ldots, 8)$ of the charge octet in the unitary symmetry scheme of Gell-Mann and Ne'eman [10-11]. In this scheme $F_{3}+\frac{1}{\sqrt{3}} F_{8}$ is the electric charge operator for the strongly interacting particles. Since the electric charge is conserved in all known interactions, the assumption $\left[e_{3}+\frac{1}{\sqrt{3}} e_{8}, P\right]=0$ is physically a very interesting one. Now by (5) and (6) it is easy to verify that $e_{3}$ is regular in $S^{C}$ (and in $S$ ) and $e_{3}, e_{8}$ span a Cartan subalgebra of $S^{C}$. But $e_{3}+\frac{1}{\sqrt{3}} e_{8}$ is not
16 Commun. math. Phys., vol.10 
regular in $S$ (or in $S^{C}$ ) since

$$
\left[e_{3}+\frac{1}{\sqrt{3}} e_{8}, e_{6}\right]=-\frac{1}{2} e_{7}+\frac{1}{2} e_{7}=0 .
$$

These remarks clarify the physical meaning of the following

Theorom 4. Suppose $S$ is a semisimple subalgebra of $G=P+S$ and let $q$ be an element of $S$ such that:

a) $q$ belongs to a Cartan subalgebra of $S^{C}$

b) $q$ has non vanishing component on each of the simple ideals of $S^{C}$. If;

$$
[q, P]=0
$$

then $G=P^{\prime} \times S$ when $P^{\prime} \approx P$. If $S$ is compact then $G=P \times S$.

Proof. We prove first that $S^{C}$ is an ideal of $G^{C}=P^{C}+S^{C}$. Let $K^{*}$ be the dual space of $K$. We define the mapping $\varrho \rightarrow h_{\varrho}$ of $K^{*}$ onto $K$ as in the proof of theorem 1 . We recall that $K^{*}$ can be equipped with a bilinear symmetric form $(\varrho, \sigma)$ such that $(\varrho, \sigma)=\varrho\left(h_{\sigma}\right)=\sigma\left(h_{\varrho}\right)$. Now let $\left\{\alpha_{1}, \alpha_{2}, \ldots, \alpha_{l}\right\}$ be a simple system of roots of $K$. We can choose the root vectors $_{ \pm \alpha_{i}}$ e so that:

$$
\begin{array}{rr}
h_{\alpha_{i}} & =\left[e_{\alpha_{i}}, e_{-\alpha_{i}}\right] \quad(i=1,2, \ldots, l), \\
{\left[h_{\alpha_{j}}, e_{\alpha_{i}}\right]} & =\left(\alpha_{i}, \alpha_{j}\right) e_{\alpha_{i}} \quad(i, j=1,2, \ldots, l) .
\end{array}
$$

The elements $e_{ \pm \alpha_{i}}$ generate $S^{C}$; hence to prove that $S^{C}$ is an ideal it suffices to show that $\left[p, e_{ \pm \alpha_{i}}\right] \in S^{C}$ for every $p \in P^{C}$. We have: $\left[q, e_{\alpha_{i}}\right]$ $\alpha_{i}(q) e_{\alpha_{i}}$ where the numbers $\alpha_{i}(q)$ can not all be zero; otherwise, since the $e_{\alpha_{i}}$ generate $S^{C}, q$ would belong to the center of $S^{C}$, but this is impossible because $q \neq 0$ and the semisimple Lie algebra $S^{C}$ has zero center. Then, without loss of generality, we can suppose $\alpha_{i}(q)=0$ for $i=1,2, \ldots, k_{1}<l$ and $\alpha_{i}(q) \neq 0$ for $i=k_{1}+1, \ldots, l$. Thus for $i>k_{1}$

$$
\left[p, e_{ \pm \alpha_{i}}\right]= \pm \frac{1}{\alpha_{i}(q)}\left[p,\left[q, e_{ \pm \alpha_{i}}\right]\right]
$$

so that by (7), (8) and the Jacobi identity:

$$
\begin{aligned}
& {\left[p, e_{ \pm \alpha_{j}}\right] \in S^{C}, \quad\left[p, h_{\alpha_{j}}\right] \in S^{C}} \\
& p \in P^{C}, \quad j=k_{1}+1, \ldots, l .
\end{aligned}
$$

Now we consider the quantities $\left(\alpha_{i}, \alpha_{j}\right), i=1,2, \ldots, k_{1}, j=k_{1}+1, \ldots, l$; these can not all be zero. In fact if this assertion is false the elements $e_{ \pm \alpha_{i}}\left(i=1,2, \ldots, k_{1}\right)$ generate a proper ideal $I$ of $S^{C}$ (reference [7] Ch. IV theorem 4). Since $\alpha_{i}(q)=0$ for $i \leqq k_{1}$ we have $[q, I]=0$. Now let $S_{1}, \ldots, S_{r}$ be the simple ideals of $S^{C}$. By hypothesis, $q=q_{1}+\cdots+q_{r}$ where $q_{k} \in S_{k}$ and $q_{k} \neq 0$. We can suppose $I=S_{1}+\cdots+S_{m}, m<r$, so that if we set $x=q_{1}+\cdots+q_{m}$, we have $x \in I, x \neq 0$ and $[x, I]=0$. This is impossible since $I$ is semisimple. Then, without loss of generality, 
we can assume:

$$
\begin{gathered}
\left(\alpha_{i}, \alpha_{j}\right)=0 \text { for } i=1, \ldots, k_{2}<k_{1} \text { and } j>k_{1}, \\
\left(\alpha_{i}, \alpha_{j}\right) \neq 0 \text { for } i=k_{2}+1, \ldots, k_{1} \text { and some } j>k_{1} .
\end{gathered}
$$

Hence for $i=k_{2}+1, \ldots, k_{1}$ we can write:

$$
\left[p, e_{ \pm \alpha_{i}}\right]= \pm \frac{1}{\left(\alpha_{i}, \alpha_{j}\right)}\left[p,\left[h_{\alpha_{j}}, e_{ \pm \alpha_{i}}\right]\right]
$$

where $j>k_{1}$. From this, by (10) and the Jacobi identity, we obtain: $\left[p, e_{ \pm \alpha_{i}}\right] \in S^{C},\left[p, h_{\alpha_{i}}\right] \in S^{C}$ for $i=k_{2}+1, \ldots, k_{1}$ and $p \in P^{C}$. At this point it is clear that by iteration of the reasoning we can conclude $\left[p, e_{ \pm \alpha_{i}}\right] \in S^{C}$ for $i=1, \ldots, l$. Thus $S^{C}$ is an ideal of $G^{C}$. Bearing in mind the remarks at the beginning of this section, it is not difficult to see that $S$ is also an ideal of $G$. From lemma II $2^{\prime}$ it follows $G=P^{\prime} \times S, P^{\prime} \approx P$. Now let $\pi$ be the projection onto $S$ with kernel $P^{\prime}$, the restriction of $\pi$ onto $L \subset P$ is a homomorphism of $L$ into $S$. Since $L$ is simple the kernel of this homomorphism is either zero or $L$. If the kernel is zero, then $\pi L \approx L$ is a non compact subalgebra of $S$. If $S$ is compact this is impossible (see Appendix) so that $\pi L=0$. Hence $L \subset P^{\prime}$ and $[L, S]=0$. Then from theorem III 1 it follows $G=P \times S$. This completes the proof.

Remark. The preceding theorem can be compared with the result stated in reference [6]. There, $q$ is assumed to be an arbitrary element of $S$. However in the proof of the statement it is implicitly assumed that any element of a (complex) simple Lie algebra can be embedded in a Cartan subalgebra. Now this does not hold in general. Consider for instance the Lie algebra $s l(2, C)$; its Cartan subalgebras are one-dimensional, so that an element of a Cartan subalgebra is necessarily regular. But $s l(2, C)$ contains a basis $\{e, f, h\}$ such that $[e, h]=2 e,[f, h]=-2 f$, $[e, f]=h$. Then $(\operatorname{ad} e)^{2} h=0$ and the Fitting null component of ad $e$ is two-dimensional. Hence $e$ is not regular and can not belong to a Cartan subalgebra.

\section{Appendix}

We recall that a compact Lie algebra is a real Lie algebra of the form $K=S \times C$, where $C$ is abelian and $S$ semisimple with a negative definite Killing form.

Let $V$ be a real (complex) vector space equipped with a bilinear symmetric (hermitian) form $\Phi$. A representation $\varrho$ of a real Lie algebra $E$ on $V$ will be called unitary if $\Phi$ is positive definite and

$$
\Phi(x, \varrho(g) y)=-\Phi(\varrho(g) x, y), \quad x, y \in V ; g \in G .
$$

With these definitions we have the following well known

Theorem. If a real Lie algebra has a unitary finitedimensional faithful representation, then it is compact. 
We can now prove the following

Corollary. Any subalgebra of a semisimple compact Lie algebra is compact.

Proof. Let $H$ be a subalgebra of the semisimple compact Lie algebra $K$. The mapping $h \rightarrow \operatorname{ad} h$ of $H$ into ad $K$ is a representation of $H$ in the vector space $K$; since $K$ has zero center this representation is faithful. The Killing form $B$ of $K$ is negative definite so that $-B$ is positive definite.

Now from the definition $B(x, y)=\operatorname{Tr} \operatorname{ad} x \operatorname{ad} y$ of the Killing form it follows:

$$
B(\operatorname{ad} a(x), y)+B(x, \operatorname{ad} a(y))=0, \quad a, x, y \in K .
$$

Hence the representation $h \rightarrow \operatorname{ad} h$ of $H$ is unitary and $H$ is compact.

Acknowledgements. The author wishes to thank Professor A. LoINGER for his kind interest, Professor F. DUIMIo and Doctor V. GorINI for valuable discussions.

\title{
References
}

1. MoGlinn, W. D.: Phys. Rev. Letters 12, 469 (1964).

2. Tomozawa, Y.: J. Math. Phys. 6, 656 (1965); Progr. Theor. Phys. 33, 319 (1965).

3. Itzykson, C.: J. Math. Phys. 6, 1331 (1965).

4. Coester, F., M. Hamermesh, and W. D. McGlinn: Phys. Rev. 135 B, 451 (1964); Mayer, M. E., H. J. Schnitzer, E. C. G. SudarShan, R. Acharya, and M. Y. HAN: Phys. Rev. 136 B, 888 (1964); BeSkow, A., and V. Ottoson: Nuovo Cimento 34, 218 (1964).

5. Ottoson, V., A. Kinlberg, and J. Nillson: Phys. Rev. 137 B, 658 (1965).

6. Sudarshan, E. C. G.: J. Math. Phys. 6, 1329 (1965).

7. JACOBSON, N.: Lie algebras. New York: Interscience 1962.

8. Helgason, Sigurdur: Differential geometry and symmetric spaces. New York: Academic Press 1962.

9. Flato, M., and D. Sternheimer: J. Math. Phys. 7, 1932 (1965).

10. GELL-MANN, M.: California Institute of Technology Syncrotron Laboratory Report $n^{0}$ CTSL-20 (1961) (unpublished).

11. Ne'eman, Y.: Nuclear Phys. 26, 222 (1961).

\author{
V. Berzi \\ Istituto di Fisica dell' Universita \\ Milano, Italia
}

